\title{
L'unité de la gastrulation chez les vertébrés
}

Juste après la gastrulation, les vertébrés possèdent des plans d'organisation similaires caractérisés par des structures embryonnaires qui se ressemblent. Parmi ces structures conservées figure la chorde, une rangée de cellules empilées le long de l'axe embryonnaire, qui est précédée dans sa partie antérieure par une structure plus large: la plaque préchordale. L'unité de la morphologie des embryons de vertébrés après la fin de la gastrulation apparaît donc clairement, et a été reconnue par les embryologistes dès le XIXe siècle $[1,2]$. Plus récemment, la conservation des patrons d'expression des gènes à homéoboîte de type Hox a permis de renouveler au niveau moléculaire l'observation de l'unité morphologique des embryons de vertébrés juste après la gastrulation, quand le plan de l'embryon se met en place [3].

En revanche, la comparaison des gastrulations des embryons de vertébrés fait d'abord ressortir des divergences. En effet, les formes générales des gastrulas de vertébrés semblent à première vue très différentes: pendant la gastrulation, les embryons de poisson et d'amphibien sont sphériques, tandis que l'embryon de poulet est aplati à la surface d'une immense vésicule vitelline et celui de souris est en forme de coupe. De plus, la présence d'une grande quantité de vitellus et/ou la nécessité de développer des structures extra-embryonnaires ont une grande influence sur le déroulement général du développement embryonnaire. Une autre différence importante entre les jeunes embryons de vertébrés est la période à laquelle les potentialités des différentes régions tissulaires se restreignent: chez les amphibiens, les potentialités d'une cellule sont, dès les premières divisions, dépendantes de sa position au sein de l'embryon, tandis que les cellules de la masse cellulaire interne de l'embryon de souris restent équivalentes jusqu'à un stade avancé.

Cependant, une série de données récentes tendent à dégager une vue unitaire de la gastrulation: les études de lignage cellulaire ont mis en évidence que les intenses mouvements morphogénétiques de la gastrulation possèdent des caractéristiques communes à tous les vertébrés. Par ailleurs, la caractérisation de nombreux gènes dont l'expression marque des populations cellulaires données pendant la gastrulation a révélé de fortes ressemblances chez des gastrulas que l'on croyait auparavant très différentes. Un nombre important de ces gènes codent pour des facteurs de transcription. Il s'agit par exemple du gène Brachyury (T) qui a été originellement cloné chez la souris [4]. Sa caractérisation a été détaillée dans l'article précédent du Lexique [5]. Un autre gène a tout d'abord été identifié chez le xénope: il s'agit du gène goosecoid $[6,7]$. Des homologues de ces gènes ont maintenant été isolés chez les principaux modèles de vertébrés. La combinaison des approches disponibles chez les différents modèles a déjà permis d'obtenir des éclairages nouveaux concernant la régulation et la fonction de ces gènes lors de la gastrulation des vertébrés.

D'autres facteurs susceptibles de régir les interactions entre les populations de cellules en mouvement ont pu être identifiés: ainsi nodal chez la souris [8] et noggin chez le xénope [9] codent pour des facteurs diffusibles. L'analyse de leur fonction constitue une première étape vers la connaissance des facteurs précoces susceptibles d'assurer les communications entre les cellules en migration de la gastrula.

\section{Des éléments nouveaux en embryologie soulignent l'unité des gastrulations de vertébrés}

Grâce au marquage de cellules uniques par injections de traceurs enzymatiques ou fluorescents, la connaissance des destins des cellules injectées avant la gastrulation a fait d'énormes progrès $[10,11]$. Chez les jeunes gastrulas d'amphibien, de poulet, de souris ou de poissonzèbre, des cartes des territoires présomptifs indiquent à quelles structures de l'embryon en construction vont appartenir les descendants d'une population de cellules occupant une région donnée de la jeune gastrula (figure 1). La comparaison des cartes des différents organismes montre qu'à une région de chaque gastrula correspond une région équivalente chez les autres gastrulas [12].

C'est essentiellement le taux et la répartition du vitellus qui sont à l'origine des différences morphologiques entre les gastrulas. Comme l'ont suggéré Beddington et Smith dans une revue récente [13], en éliminant virtuellement les vésicules vitellines du pleurodèle (un batracien modèle) et du poisson-zèbre et en faisant se rejoindre par une projection fictive les bords de la lèvre du blastopore ou de la marge du blastoderme de ces deux espèces, les ressemblances entre les gastrulas de ces vertébrés inférieurs et celles des amniotes (poulet, souris) deviennent très claires (figure 2 ). Ainsi, chez le pleurodèle, la région située à proximité de la lèvre du blastopore comprend les précurseurs du mésoderme. Des régions homologues sont situées chez la souris et le poulet au bord de la ligne primitive, un sillon dans lequel les cellules $\mathrm{m} / \mathrm{s} n^{\circ} 1$ vol. 10, janvier 94 
s'engouffirent pour participer à la formation du mésoderme. Chez le poisson-zèbre, l'équivalent de ces domaines se trouve à la marge du blastoderme, à l'endroit où les cellules s'enroulent vers l'intérieur.

Des similitudes existent donc entre les cartes des destins cellulaires des jeunes gastrulas de vertébrés, ce qui conforte l'idée que, malgré une apparente diversité du déroulement de la gastrulation, les signaux actifs lors de ce processus doivent être conservés.

\section{Diversité des stratégies d'isolement des gènes exprimés pendant la gastrulation}

Chez les vertébrés, diverses stratégies ont conduit à identifier les premiers acteurs moléculaires de la gastrulation. Un premier type d'approche tire partie de la conservation au cours de l'évolution des motifs présents parmi les gènes de développement de drosophile. Un de ces motifs est l'homéoboîte [14]. Afin de cloner des gènes exprimés pen- dant la gastrulation, des séquences correspondant à une région ou à la totalité de certaines homéoboîtes ont été utilisées lors d'expériences de PCR et de criblage de banques d'ADNc construites à partir de gastrulas. Les gènes isolés par cette démarche font par exemple partie des familles des gènes à homéoboîtes caudal, even-skipped ou fork-head de drosophile. Ainsi, Xnot [15] et $X \lim$ [16], deux gènes spécifiques de l'organisateur de xénope aux homéoboîtes très divergentes, ont aussi été découverts. Un autre gène spécifique de l'organisateur, goosecoid, a été isolé en suivant une voie de ce type. Blumberg et al. ont conçu un oligonucléotide dégénéré correspondant à l'hélice III de l'homéoboîte [6]. Ce domaine dont la conservation est extraordinairement forte correspond au domaine principal de liaison à l'ADN. En criblant avec un tel oligonucléotide une banque d'ADNc construite à partir d'explants de lèvre dorsale de blastopore de xénope, l'équipe de E. De Robertis a cloné plusieurs gènes dont goosecoid [6].

Un second type d'approche consiste à isoler des gènes impliqués dans un

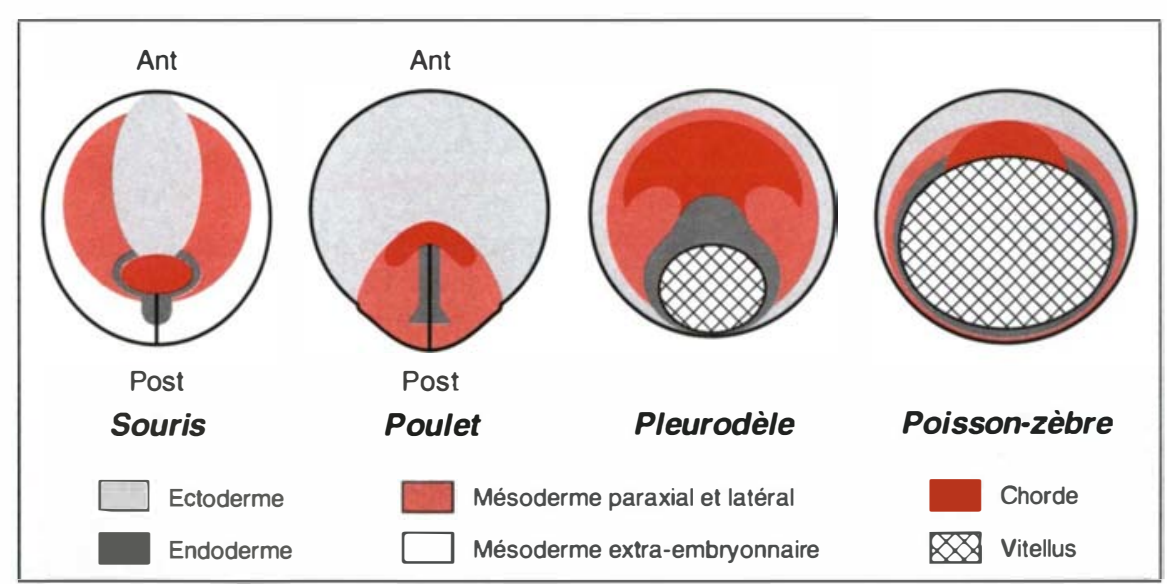

Figure 1. Carte des destinées présomptives aux stades précoces de la gastrulation de la souris, du poulet, du pleurodèle et du poissonzèbre. Les chevauchements entre les différents domaines, repérés lors des études de lignage, ne sont pas représentés sur ce schéma. L'épiblaste de la souris a été aplati. Les lignes primitives de la souris et du poulet sont schématisées par un trait. Les embryons de pleurodèle et de poisson-zèbre sont représentés par une vue dorsale en perspective (d'après [12, 42-44]). $m / s n^{\circ} 1$ vol. 10 , janvier 94 processus particulier selon des critères fonctionnels. Ainsi, le clonage de noggin a été effectué par l'intermédiaire d'un test basé sur le déclenchement d'un programme de développement de type dorsal à la suite d'injections de messagers transcrits in vitro dans un œuf [9]. Cette propriété a été révélée en restaurant l'apparition de structures dorsales chez des embryons de xénope ventralisés par des irradiations aux UV. La mise en ouvre d'une telle stratégie de clonage chez la souris est plus délicate en raison de la difficulté de manipulation des embryons de mammifères.

Deux démarches qui tirent parti de l'analyse génétique ont en revanche abouti à l'isolement de deux gènes de souris. La première est le clonage positionnel de la mutation Brachyury [4] qui constitue l'aboutissement d'un long travail (voir $\mathrm{m} / \mathrm{s}$ $n^{\circ} 10$, vol. 9, p. 1118). La seconde démarche, utilisant la mutagenèse insertionnelle, a débuté par l'établissement d'un stock de souris transgéniques portant des insertions multiples d'un vecteur rétroviral MPSVnéo [17]. Pour l'une de ces insertions, la gastrulation d'embryons homozygotes se déroule de manière anormale et le gène inactivé par cette insertion a pu être cloné et a été nommé nodal [8]. La diversité des stratégies mises en œuvre pour isoler des gènes exprimés pendant la gastrulation montre que plusieurs voies peuvent être empruntées. Actuellement, l'essor de la mutagenèse insertionnelle dans des cellules ES de souris [18, 19] et des approches mutationnelles chez le poisson-zèbre [20] devraient permettre de caractériser de nouveaux gènes impliqués dans la gastrulation.

\section{Les facteurs de transcription exprimés au début de la gastrulation}

Lors de la gastrulation, la spécification des principales populations cellulaires s'accompagne du déclenchement de programmes génétiques particuliers comprenant, notam- 
ment, l'activation d'un ensemble unique de facteurs de transcription. Récemment, plusieurs gènes s'exprimant au cours de la gastrulation et codant pour des protéines possédant un motif de liaison à l'ADN de type doigts de zinc ou homéodomaine ont été isolés. Ainsi, les gènes turist et snail, clonés chez la souris et le xénope, sont activés dans des territoires mésodermiques, selon un profil compatible avec une implication possible de ces gènes dans la spécification de sous-populations de cellules mésodermiques [21, 22]. C.es dernières années, l'accumulation de données recueillies chez plusieurs espèces a permis de cerner plus précisément le rôle de deux facteurs de transcription : Brachyury (T) et goose coid.

\section{Brachyury (T)}

Chez la souris, l'expression de $T$ débute dans l'ectoderme et le mésoderme qui bordent la ligne primitive [23]. Au cours de la gastrulation, l'expression se maintient dans la région de la ligne primitive ainsi que dans les précurseurs de la chorde. Des homologues de $T$ ont été isolés chez le xénope et le poisson-zèbre. L'expression du gène $T$ de xénope, nommé Xbra, est fortement activée dans toute la région marginale de la blastula tardive, le stade embryonnaire qui précède le déclenchement de la gastrulation [24], et les cellules de cette région qui s'engouffrent à l'intérieur de l'embryon cessent d'exprimer $T$, à l'exception de celles qui participent à la formation de la chorde. Schulte-Merker et al. ont montré que le patron d'expression du gène $T$ de poisson-zèbre, nommé $Z f-T$ puis $n l l$, ressemble à celui des gènes de souris et de xénope [25]: les messagers et la protéine sont localisés au bord du blastoderme, dans une région présomptive du mésoderme; plus tard, l'expression ne persiste que dans la chorde et dans une masse de cellules postérieures qui prennent part à la morphogenèse de la queue lors d'un phase de développement qui suit la gastrula-

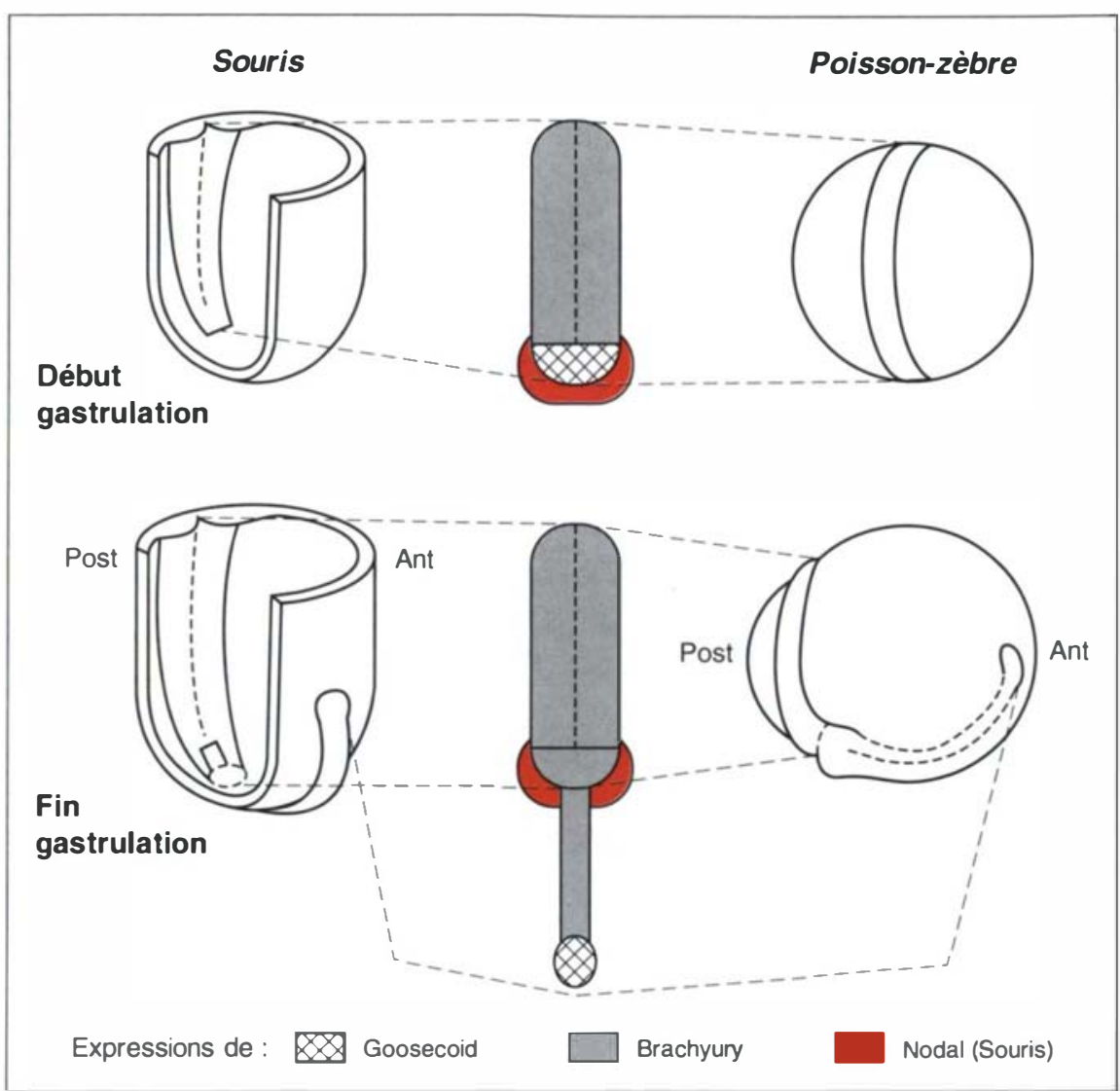

Figure 2. Diagrammes représentant les gastrulas de souris (à gauche) et de poisson-zèbre (à droite) au début (en haut) et à la fin (en bas) de la gastrulation. En haut, au centre, sont schématisées les projections fictives de la région de la ligne primitive de la souris, et de la marge du blastoderme du poisson-zèbre, étirée puis fusionnée latéralement. En bas, au centre, ont été ajoutées les projections des précurseurs de la chorde et de la plaque préchordale. La schématisation des domaines d'expression des gènes Brachyury et goosecoid sur les diagrammes centraux souligne la similitude des profils d'expression des homologues de souris et de poisson-zèbre (d'après [13]).

\section{Goosecoid}

La caractéristique la plus captivante des patrons d'expression de goosecoid s'observe aux stades qui précèdent la gastrulation. Chez le poulet, une population de cellules positives pour goosecoid peut être identifiée avant même que la ligne primitive ne se forme. Ces cellules peuplent un domaine postérieur de l'embryon, et les analyses clonales montrent qu'elles migrent vers le noud de Hensen [26]. Chez le poisson-zèbre, les messagers goosecoid sont d'abord répartis ubiquitairement dans la blastula [27], puis, juste avant la gas- trulation, ils ne sont plus détectés que dans la région marginale du blastoderme.

Au début de la gastrulation, différents vertébrés est restreinte à des structures homologues, connues sous le nom d'organisateur chez les amphibiens [7, 28], de nœud de Hensen chez les amniotes [26], ou de bouclier embryonnaire chez le poisson-zèbre [27]. I.es populations de cellules marquées positivement par les gènes goosecoid subissent d'importantes migrations au cours de la gastrulation. Dans les diffél'expression du gène goosecoid des 
rents embryons, les cellules quittent les régions citées ci-dessus, pour participer, chez tous les vertébrés où le gène a été caractérisé, à la formation des cellules mésodermiques antérieures de la plaque préchordale. Chez les vertébrés, goosecoid marque donc une population de cellules en mouvement destinée à participer aux structures céphaliques mésodermiques et endodermiques.

\section{Quelles fonctions pour ces gènes?}

C'est la fonction du facteur de transcription codé par le gène $T$ qui peut être cernée le plus précisément puisque des tests fonctionnels ont été développés chez la souris et le xénope, et que des allèles nuls de cette mutation sont depuis longtemps caractérisés chez la souris [5, 29]. Récemment, des allèles nuls du gène $T$ de poisson-zèbre ont été décrits [30], ce qui a permis d'obtenir de nouvelles données sur la fonction de ce gène.

Bien que $T$ soit exprimé dans tous les précurseurs du mésoderme au cours de la gastrulation, la présence de son produit ne semble directement nécessaire qu'à la seule mise en place de la chorde et de la queue. Le mode d'action de $T$ dans la morphogenèse postérieure reste largement inconnu. On a longtemps cru que la formation de la queue était secondairement perturbée par l'absence de chorde différenciée. Il semble en fait que $T$ ait un rôle spécifique dans le bourgeon caudal où il est exprimé [13]. En effet, lorsque $X b r a$, le gène $T$ de xénope, est surexprimé par injection de messagers dans l'œu de xénope, une différenciation ectopique de mésoderme, mais aussi le développement fréquent d'une seconde queue, sont observés [31]. Par ailleurs, $T$ active la transcription d'autres familles de gènes codant pour des facteurs de transcription spécifiques de la région caudale. Ainsi, la surexpression de Xbra dans des explants cellulaires en culture provoque une activation ectopique de Xhox3, un gène à homéoboîte de la famille evenskipped, normalement exprimé dans la région postérieure [31]. Chez le $m / s n^{\circ} 1$ vol. 10 , janvier 94 poisson-zèbre, les messagers d'un autre gène de type even-skipped, dont l'expression est normalement caudale pendant la somitogenèse, ne sont plus détectés à ce stade dans un embryon homozygote $T / T$ [32]. La chorde est une source importante de signaux intercellulaires qui contribuent, dans la région troncale, à la polarisation du tube nerveux et des somites. Il est donc particulièrement intéressant que Halpern, et Kimmel aient récemment montré, chez le poisson-zèbre, que chez les mutants $T / T$, les précurseurs de la chorde conservent certaines capacités inductrices tandis que d'autres sont altérées. En effet, des précurseurs $T / T$ sont capables, à l'instar des précurseurs de type sauvage, d'induire une rangée de cellules située à la base du tube nerveux, le " plancher ". En revanche, la chorde ne possède plus les capacités d'induire un autre type cellulaire spécialisé situé dans la région médiane des somites [30].

Chez la souris, certaines données mènent à l'idée que l'absence de $T$ provoque une anomalie de migration des précurseurs de la chorde au cours de la gastrulation [33]. En effet, si des cellules ES murines, mutantes pour $T$, sont placées dans un embryon receveur de type sauvage, elles ne subissent pas les intenses mouvements d'extension convergente caractéristiques de la chorde et restent majoritairement dans la région postérieure de l'embryon [33]. Une série d'observations récentes obtenues par le laboratoire de Kimmel sur le poisson-zèbre a permis, grâce en particulier à la transparence et à la grande taille de l'embryon, de mettre en évidence que des précurseurs de la chorde migrent de manière appropriée dans l'embryon homozygote mutant pour le gène $T[30]$. C'est seulement à la fin de la gastrulation que leur différenciation semble incorrecte : au lieu d'être de grande taille et de posséder une forme caractéristique due à une vacuolisation, les cellules $T / T$ ne se distinguent pas du mésoderme paraxial. Elles restent de petite taille et d'aspect mésenchymateux. Un mauvais positionnement des cellules de la chorde lié à des anomalies de migration pendant la gastrulation ne serait donc sans doute pas la cause principale des défauts observés chez les embryons $T / T$ de poisson-zèbre.

En revanche, un exemple très clair de régulation de la migration cellulaire sous le contrôle d'un facteur de transcription a été mis en évidence par l'équipe de De Robertis [34]. Celle-ci a montré que l'expression ectopique de messagers goosecoid dans un groupe de blastomères les conduit à migrer vers la région antérieure de l'embryon. De plus, ces blastomères migrent d'une façon active, caractéristique des cellules de la plaque préchordale, oì s'exprime normalement goosecoid. Il semble donc que la présence de goosecoid dans un groupe de cellules influe sur le destin de ces cellules en déclenchant la machinerie cellulaire à l'origine d'un comportement migratoire caractéristique.

\section{Facteurs diffusibles exprimés au cours de la gastrulation}

Ces dernières années, de nombreuses substances diffusibles impliquées dans le phénomène d'induction mésodermique ont pu être identifiées chez le xénope [35]. Il s'agit principalement de membres des familles TGF- $\beta$, FGF et W'nt [36]. Plusieurs des gènes codant pour ces facteurs ont été clonés chez d'autres vertébrés mais les différents phénomènes d'induction liés à ces facteurs restent moins bien compris chez ces espèces.

Chez l'amphibien, les données apportées par les expériences d'embryologie classique, combinées à celles plus récentes utilisant l'injection de protéines purifiées ou l'injection d'ARNm correspondant, ont permis de dresser un plan d'organisation générale des processus d'induction en œuvre lors du développement précoce (figure 3 [37, 38]). Dans la blastula, une première série de signaux induisent la formation de mésoderme de type ventral ou dorsal. Dans la gastrula, la régionalisation dorso-ventrale du méso- 


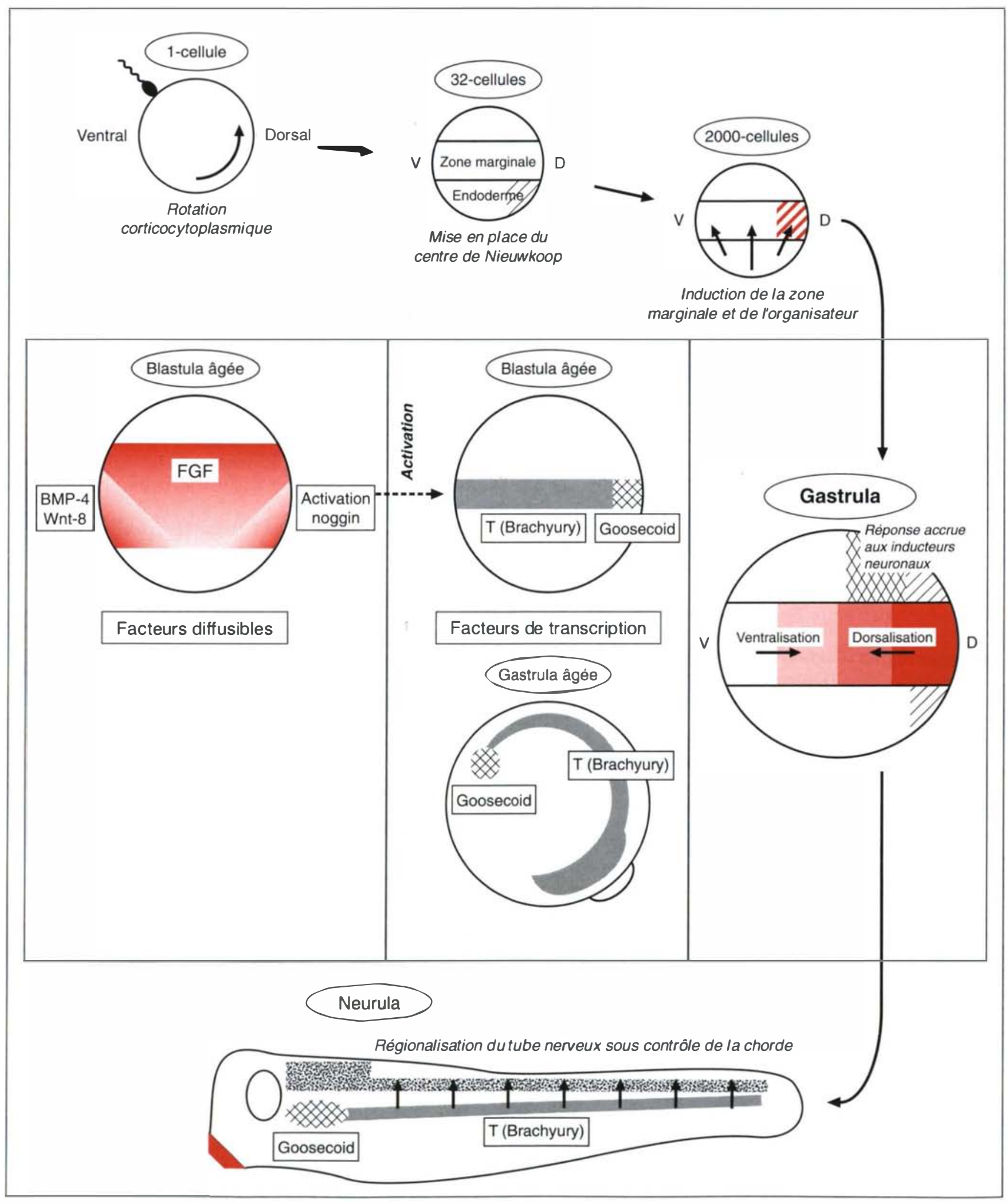


derme est affinée sous l'influence d'interactions ventralisantes ou dorsalisantes qui prennent place dans un contexte de mouvements morphogénétiques complexes (figure 3). Deux gènes clonés récemment et qui codent pour des facteurs diffusibles, noggin et nodal, suscitent un intérêt particulier car tous deux sont exprimés au cours de la gastrulation dans la région organisatrice, une région connue de longue date pour ses importantes capacités inductrices.

\section{noggin}

Chez le xénope, le transcrit zygotique du gène noggin est localisé dans la zone marginale dorsale de la blastula tardive, et dans la partie dorsale de la lèvre du blastopore pendant la gastrulation. Après invagination, les cellules positives pour noggin sont situées dans les précurseurs de la chorde et de la plaque préchordale [9].

Noggin partage un effet "dorsalisant " avec deux protéines codées par des gènes de la famille Wnt: Wntl et Wnt8. Cependant, seul noggin possède des propriétés laissant supposer qu'il pourrait faire partie du signal "dorsalisant " réellement actif au cours de la gastrulation. Premièrement, au contraire des gènes Wntl et Wnt 8 qui sont exprimés soit trop tard, soit au mauvais endroit, noggin est exprimé dans la région dorsale de l'embryon selon un patron compatible avec sa fonction dorsalisante; de plus, l'incubation de cellules de la zone marginale ventrale (cellules précurseurs du mésoderme ventral) avec la protéine Noggin purifiée provoque l'apparition de marqueurs spécifiques du mésoderme dorsal [39].

Par ailleurs, des résultats récents suggèrent qu'à la fin de la gastrulation, noggin pourrait être un élément important du signal d'induction neurale. noggin est exprimé dans la chorde dont les propriétés d'induction neurale sont connues depuis longtemps. L'incubation d'ectoderme avec la protéine Noggin purifiée induit l'apparition de tissus nerveux en absence de mésoderme dorsal. Cependant, noggin ne serait qu'un des constituants du signal d'induction neural car il n'induit que l'apparition de marqueurs de structures nerveuses antérieures, tel ot $x A[40]$.

\section{nodal}

Le gène nodal, qui vient d'être cloné chez la souris, code pour une protéine de la famille du TGF- $\beta$ [8]. Son expression est restreinte dans le temps et dans l'espace. Faiblement transcrit au cours des étapes précoces de la gastrulation, nodal n'est détectable par hybridation in situ qu'à partir de 7,5 jours de développement. Le patron d'expression du gène nodal est particulièrement inté- ressant car les cellules positives pour nodal sont localisées au niveau du noud pendant toute la gastrulation. Les cellules cessent d'exprimer nodal de manière concomitante à la disparition de celui-ci.

Plusieurs données suggèrent que nodal a un rôle essentiel dans la formation du mésoderme chez la souris. Les embryons homozygotes pour une insertion rétrovirale dans le gène nodal ne forment pas de mésoderme [17]. Après implantation, ces embryons possèdent des structures extra-embryonnaires bien développées alors que l'ectoderme embryonnaire prolifère puis dégénère rapidement. Des cellules ES homozygotes pour cette insertion rétrovirale sont capables, lorsqu'elles sont injectées dans un blastocyste normal, de participer à la formation de tous les tissus; en particulier, elles se différencient normalement en mésoderme [41]. La protéine Nodal n'est donc pas nécessaire, de façon cellulaire autonome, à la différenciation des cellules mésodermiques, mais plus probablement elle constituerait un signal sécrété par les cellules du nœud agissant sur les cellules en cours d'invagination et déterminant alors leur participation au lignage mésodermique.

\section{Conclusion}

Nodal et Noggin sont deux facteurs synthétisés et sécrétés par les cellules du centre organisateur, importants dans la mise en place du plan d'organisation générale de l'embryon. Jusqu'à présent, noggin n'a été isolé que chez le xénope et nodal que chez la souris; des gènes analogues devraient rapidement être clonés chez les autres espèces de vertébrés et il est probable que leur analyse renforcera l'idée d'une importante conservation des gènes exprimés pendant la gastrulation, montrée par l'étude de $T$ et goosecoid. Une unité encore plus précoce du développement des vertébrés existe-t-elle? L'étude des gènes régulant l'expression de $T$ et goosecoid pourrait fournir des éléments de réponse à cette question.

De plus, l'utilisation de différentes 
stratégies disponibles chez la souris et le poisson-zèbre devrait mener à l'isolement d'un nombre croissant de facteurs impliqués dans la gastrulation. L'analyse fonctionnelle de ces facteurs, menée essentiellement chez l'amphibien, devrait dans l'avenir s'enrichir de résultats issus des expériences réalisées chez les autres modèles de vertébrés

\section{Jean-Stéphane Joly \\ Michel Cohen-Tannoudji}

Unité de génétique des mammiferes, institut Pasteur, 25, rue du Docteur-Roux, 75015 Paris, France.

\section{RÉFÉRENCES}

1. Haeckel E. Histoire de la création des êtres organisés. Paris: Alfred Costes, 1992.

2. Von Baer KE. Entwicklungsgeschichte der Thiere: Beobachtung und Reflexion. Kônigsberg : Bornträger, 1828.

3. Jacob F. L'unité du vivant. médecine/sciences $1990 ; 6$ : 222-7.

4. Hermann BG, Labeit S, Poutska A, King TR, Lehrach $H$. Cloning of the $T$ gene required in mesoderm formation in the mouse. Nature 1990; 343: 617-22.

5. Camus A, Babinet C. Brachyury, un gène essentiel pour la gastrulation et la formation du mésoderme chez les vertébrés. médecine/sciences 1993; 9: 1118-21.

6. Blumberg B, Wright CVE, De Robertis EM, Cho KWY. Organizer-specific homeobox genes in Xenopus laevis embryos. Science 1991; 253 : 194-6.

7. Cho KWY, Blumberg B, Steinbesser $\mathrm{H}$ De Robertis E. Molecular nature of the Spemann's organizer: the role of the Xeno pus homeobox gene goosecoid. Cell 1991; $67: 1111-20$

8. Zhou X, Sasaki H, Lowe L, Hogan BLM, Kuehn MR. nodal is a novel TGF- $\beta$ like gene expressed in the mouse node during gastrulation. Nature 1993; 361: 543-7.
9. Smith W'C, Harland RM. Expression cloning of noggin, a new dorsalizing factor localized to the Spemann organizer in Xenopus embryos. Cell 1992 ; 70 : 829-40.

10. Condamine H. L'analyse clonale. méde cine/sciences 1991; 7 : 729-31.

11. Condamine H. Les enseignements de l'analyse clonale. médecine/sciences 1991; 7 : 855-8.

12. Stern CD. Mesoderm induction and development of the embryonic axis in amniotes. Trends Genet 1992; 8: 158-63.

13. Beddington RSP, Smith JC. Control of vertebrate gastrulation: inducing signals and responding genes. Cur Biol 1993; 3 : 655-61.

14. Scott MP, Tamkun JW, Hartzell GW The structure and function of the homeodomain. Biochem Biophys Acta 1989; 989 25-48.

15. Von Dassow G, Schmidt JE, Kimelman D. Induction of the Xenopus organizer expression and regulation of Xnot, a nove FGF and activin-regulated homeo box gene. Genes I)ev 1993; 7 : 355-66.

16. Taira M, Jamrich M, Good PJ, Dawid IB. The LIM domain-containing homeo box gene Xlim-l is expressed specifically in the organizer region of Xenopus gastrula embryos. Genes Dev 1992; 6: 356-66.

17. Ianaccone PM, Zhou X, Khokha $M$ Boucher D, Kuehn MR. Insertional mutation of a gene involved in growth regulation of the early mouse embryo. Dev Dynam 1992 ; 194 : 198-208.

18. Babinet C. Les cellules souches embryonnaires de souris: une voie privilégiée de transformation génétique à l'échelle animale. médecine/sciences 1992; 8 268-75.

19. Joyner A, Auerbach A, Skarnes WC. The gene trap approach in embryonic stem cells: the potuntial for genetic screens in mice. In : Post-implantation development in the monse. Ciba Foundation Symposium 165. Chichester: John Wiley \& Sons.

20. Mullins MC, Nũsslein-Vohlard C. Mutational approaches to studying embryonic pattern formation in the zebrafish. Curr OP Genet Dev 1993; 3 : 648-54.

21. Sargent MG, Bennett MF. Identification in Xenopus of a structural homologue of the Drosophila gene snail. Development 1990 ; 109 : 967-73.

22. Smith DE, Franco del Amo F, Gridley T. Isolation of Sna, a mouse gene homologous to the Drosophila genes snail and escangot: its expression pattern suggests multiple roles during post-implantation deve lopment. Development 1992; 116 : 1033-9.

23. Hermann BG. Expression pattern of the Brachyury gene in whole-mount TWIS/TWIS mutant embryos. Development 1991 ; 113 : 913-7.

24. Smith JC, Price BJM, Green JBA, Weigel D, Hermann BG. Expression of a Xeno pus homolog of Brachyury (T) is an imme diate early response to mesoderm induction. Cell 1991; 67 : 79-87.

25. Schulte-Merker S, Ho RK, Hermann BG, Nusslein-Volhard C. The protein product of the zebrafish homologue of the mouse $T$ gene is expressed in nuclei of the germ ring and the notochord of the early embryo. Development 1992; 116 : 1021-32.

26. Izpisua-Belmonte JC, De Robertis EM, Storey KG, Stern CD. The homeobox gene goosecoid and the origin of organizer cells in the early chick blastoderm. Cell 1993 $74: 645-59$

27. Stachel SE, Grunwald DJ, Myers PZ Lithium perturbation and goosecoid expression identify a dorsal specification pathway in the pregastrula zebrafish. Development 1993 ; $117: 1261-74$

28. Condamine $\mathrm{H}$. L'organisateur de l'embryon d'amphibien. médecine/sciences $1992 ; 8$ : 483-6.

29. Chesley P. Development of the short tailed mutation in the house mouse. $J$ Exp Zool 1935; 70 : 429-59.

30. Halpern ME, Ho RK, Walker C, Kimmel CB. Induction of muscle pioneers and floor plate is distinguished by the zebrafish no tail mutation. Cell 1993; 75 : 99-111.

31. Cunliffe V, Smith IC. Ectopic mesoderm formation in Xenopus embryos caused by widespread expression of a Brachyury homologue. Nature 1992; 358: 427-30.

32. Joly JS, Joly C, Schulte-Merker S, Boulekbache $H$, Condamine $H$. The ventral and posterior expression of the zebrafish homeobox gene evel is perturbed in dorsalized and mutant embryos. Development 1994 (sous presse).

33. Wilson V, Rashbass P, Beddington RSP. Chimeric analysis of $T$ (Brachyury) gene function. Development 1993; 117: 1321-33.

34. Niehrs C, Keller R, Cho KWY, De Robertis EM. The homeobox gene goosecoid controls cell migration in Xenopus embryos. Cell 1993; 72 : 491-503.

35. Condamine $H$. Induction mésodermique et dorsalisation. médecine/sciences 1992 ; 8: $379-81$. 
36. Smith JC. Mesoderm-inducing factors in early vertebrate development. $E M B O J$ 1993 ; 12 : 4463-70.

37. Woodland HR. Identifying the three signals. Curr Biol 1993; 3 : 27-9.

38. Sive HL. The frog prince-ss : a molecular formula for dorsoventral patterning in Xenopus. Genes Dev 1993; 7: 1-12.

39. Smith WC, Knecht AK, Wu M, Harland RM. Secreted noggin protein mimics the Spemann organizer in dorsalizing Xeno pus mesoderm. Nature 1993; 361 : 547-9.

40. Lamb TM, Knecht AK, Smith WC, Stachel SE, Economides AN, Stahl N, Yancopoulos GD, Harland R. Neural induction by the secreted polypeptide noggin. Science 1993 ; 262 : 713-8.

41. Conlon FL, Barth KS, Robertson EJ. A novel retrovirally induced embryonic lethal mutation in the mouse : assessment of the developmental fate of embryonic stem cells homo ygous for the 413.d proviral integration. Development 1991; 111: 969-81.

42. Lawson KA, Meneses II, Pedersen RA. Clonal analysis of epiblast fate during germ layer formation in the mouse embryo. Deve lopment 1991; 113 : 891-911.

43. Delarue M, Sanchez S, Johnson KE, Darribere T, Boucaut JC. A fate map of superficial and deep circumblastoporal cells in the early gastrula of Pleurodeles waltl. Development 1992; 114: 135-46.

44. Kimmel CB, Warga RM, Schilling TF. Origin and organisation of the zebrafish fate map. Development 1989; 108 : 581-94.

45. Howard JE, Smith JC. Analysis of gastrulation: different types of gastrulation movemens are induced by different mesoderm-inducing factors in Xenopus laevis. Mech Ageing Dev 1993; 43: 37-48.

\section{TIRÉS A PART}

J.-S. Joly.

\section{ERRATUM}

Il a été omis d'indiquer que le cliché de la figure 1 du Lexique embryologie $\left(\mathrm{m} / \mathrm{s} n^{\circ} 10\right.$, vol. 9, p. 1120) provenait du Dr Rosa Beddington; les auteurs s'excusent de cette omission et la remercient de leur avoir fourni ce cliché. 